\title{
miR-454 promotes survival and induces oxaliplatin resistance in gastric carcinoma cells by targeting CYLD
}

\author{
CHAOQUN HUANG ${ }^{1-3^{*}}$, JIUYANG LIU $^{1-3^{*}}$, XUEKAI PAN ${ }^{1-3}$, CHUNWEI PENG ${ }^{1-3}$, \\ BIN XIONG ${ }^{1-3}$, MAOHUI FENG ${ }^{1-3}$ and XIAOJUN YANG ${ }^{1-3}$ \\ ${ }^{1}$ Department of Gastrointestinal Surgery, Zhongnan Hospital of Wuhan University; \\ ${ }^{2}$ Hubei Cancer Clinical Study Center and Hubei Key Laboratory of Tumor Biological Behaviors; \\ ${ }^{3}$ Wuhan Clinical Research Center for Peritoneal Carcinomatosis, Wuhan, Hubei 430060, P.R. China
}

Received July 19, 2018; Accepted October 9, 2019

DOI: $10.3892 / \mathrm{etm} .2020 .8655$

\begin{abstract}
MicroRNA-454 (miR-454), is involved in the progression of various types of cancers. The present study aimed to evaluate the effect of miR-454 on the progression of gastric cancer. SGC-7901 cells overexpressing or silencing miR454 were constructed via transfection and the survival rate of the cells was determined. The relationship between miR-454 and cylindromatosis (CYLD) was explored and the influence of $\mathrm{miR}-454$ on oxaliplatin resistance was investigated in SGC-7901 cells. It was determined that overexpression of miR-454 increased the number of colonies and reduced apoptosis rate of SGC-7901 cells. The CYLD gene was identified as a direct target of miR-454. miR-454 overexpression downregulated the expression of CYLD, leading to an increase in SGC-7901 cell proliferation. Finally, miR-454 was also demonstrated to induce resistance to oxaliplatin in gastric cancer cells. In conclusion, the present in vitro findings suggested that miR-454 might be a novel therapeutic target for gastric cancer.
\end{abstract}

\section{Introduction}

Gastric cancer commonly develops within the lining of the stomach (1). The diagnosis rate has recently greatly increased with 28,000 new cases of gastric cancer diagnosed in the USA during 2017 according to the National Cancer Institute (2). The mechanism for the development and progression of gastric cancer is not fully understood which hampers the search for novel treatments (3). Chemotherapy and radiotherapy are the most commonly used approaches to treat gastric cancer

Correspondence to: Dr Xiaojun Yang, Department of Gastrointestinal Surgery, Zhongnan Hospital of Wuhan University, 169 Donghu Road, Wuhan, Hubei 430060, P.R. China

E-mail: xiaoJunYangzxc@163.com

${ }^{*}$ Contributed equally

Key words: miR-454, cylindromatosis, SGC-7901 cells, oxaliplatin despite being time-consuming and susceptible to development of drug resistance (4). The mechanisms of gastric cancer were reported to involve the increasing platinum-DNA adducts and apoptosis-regulating genes (5). It is of great value to understand the underlying mechanisms to accelerate the search for a new therapeutic target for gastric cancer.

MicroRNAs (miRs) are a class of small, single-stranded and non-protein-coding RNAs. They are implicated in the expression of mRNA (6) and regulation of various biological activities such as tumor development, cardiac diseases and hepatitis (7). Recent studies have demonstrated that miRs facilitated cancer progression by targeting specific genes $(8,9)$ and some functional miRs may be used to treat cancer. A common type of miR, miR-454, has been identified to be involved in the development of cancer (10). Expression of miR-454 is downregulated in osteosarcoma and glioblastoma and thus recognized as a potential tumor suppressor $(11,12)$. By contrast, miR-454 is overexpressed in colorectal cancer, uveal melanoma and hepatocellular carcinoma suggesting that it could also be an oncogene $(13,14)$. However, the relationship between miR-454 and gastric cancer remains uninvestigated.

The present study demonstrated that miR-454 promoted cellular proliferation and inhibited apoptosis of SGC-7901 gastric carcinoma cells, through downregulation of CYLD. It was identified that miR-454 could also induce oxaliplatin (OXA) resistance of gastric carcinoma cell via targeting CYLD. Taken together, the results suggested that miR-454 may be a potential novel therapeutic target of gastric cancer.

\section{Materials and methods}

Cell culture and transfection. Human SGC-7901 gastric cancer cells were obtained from the Cell Bank of the Guangzhou Institute of Biochemistry and Cell Biology (Guangzhou, China). The cells were cultured in Dulbecco's modified Eagle's medium (DMEM), supplemented with fetal bovine serum (FBS) and incubated at $37^{\circ} \mathrm{C}$ in a humidified atmosphere containing $5 \% \mathrm{CO}_{2}$.

The control (scramble) and miR-454 mimics, and the miR-454 inhibitor and its associated negative control, were synthesized by Shanghai GenePharma Co., Ltd. and added to 
cells at a concentration of $20 \mathrm{nmol} / \mathrm{l}$ per oligonucleotide. The sequences were as follows: miR-454 mimic, 5'-UGGGAUAUU CGCUGGAAUCCUCAU-3'; miR-454 inhibitor, 5'-UCACAU AGGAAUAAAAAGCCAUA-3'; inhibitor control, 5'-CAG UACUUUUGUGUAGUACAA-3'. Cells were collected $48 \mathrm{~h}$ post-transfection for subsequent experimentation. DharmaFECT1 reagent (GE Healthcare Dharmacon, Inc.) was used to perform the transfections according to manufacturer's protocol.

For the ectopic expression of CYLD, CYLD ORFs with 3'-UTR was amplified from the RNA samples of HepG2 or BEL-7402 cells using PCR and subcloned into the two restriction sites of pEGFP-C1 (Clontech Laboratories, Inc.) and pGL3 vectors (Promega Corporation). The primers used were as follows: CYLD-3'UTR-GFP forward, 5'-GCCCTC GAGCTTGACTCCGTTCCCCTTCAGAC-3' and reverse, 5'-GCCGGATCCAACCAAGGGCAGTTGAGTC-3' and CYLD-3' UTR-luc forward, 5'-GCCCCGCGGCTCCGTTCC CCTTCAGAC-3' and reverse, 5'-GCCCTGCAGAACCAA GGGCAGTTGAGTC-3'. Reporter gene assay was initiated $24 \mathrm{~h}$ post-transfection. A total of $5 \mu \mathrm{g}$ plasmids were used per transfection reaction. Lipofectamine ${ }^{\circledR} 3000$ transfection reagent was used in this study (Thermo Fisher Scientific, Inc.) according to the manufacturer's protocol.

Reverse transcription-quantitative polymerase chain reaction $(R T-q P C R)$. Total RNA was isolated using TRIzol ${ }^{\circledR}$ Reagent (Invitrogen; Thermo Fisher Scientific, Inc.). cDNA was synthesized using High-Capacity cDNA reverse transcriptase kit (Thermo Fisher Scientific, Inc.) according to the manufacturer's protocol. The expression of mRNA and miRNA were quantified using TaqMan ${ }^{\mathrm{TM}}$ Universal PCR Master Mix (Invitrogen; Thermo Fisher Scientific, Inc.) by RT-qPCR in the ABI 7500 System (Bio-Rad Laboratories, Inc.) according to manufacturer's protocols. The primers selected are as follows: CYLD forward, 5'-TCCTCTCCAAAATGCCAGAG-3' and reverse, 5'-GGCGGATTGGAAATGAACTT-3'; miR-454 forward, 5'-TCAAGAGGCGAACACACAAC-3' and reverse, 5'-GGC CTTTTCATTGTTTTCCA-3'; GAPDH forward, 5'-GACTCA TGACCACAGTCCATGC-3' and reverse, 3'-AGAGGCAGG GATGATGTTCTG-5' and U6 snRNA forward, 5'-ATACAG AGAAAGTTAGCACGG-3' and reverse, 3'-GGAATGCTT CAAAGAGTTGTG-5'. GAPDH and U6 snRNA were selected as the internal control for mRNA and miRNAs, respectively. The thermocycling conditions were as follows: Initial denaturation at $95^{\circ} \mathrm{C}$ for $20 \mathrm{sec}$, followed by 45 cycles of $95^{\circ} \mathrm{C}$ for $10 \mathrm{sec}$ and $70^{\circ} \mathrm{C}$ for $5 \mathrm{sec}$. Relative expression levels were calculated using the $2^{-\triangle \Delta \mathrm{Cq}}$ method (15).

Cell proliferation assay. SGC-7901 cancer cells were seeded in 96-well plates (4x10 ${ }^{3}$ cells/well). CCK-8 solution (Dojindo Molecular Technologies, Inc.) was added to each well at 24, 48, 72,96 and $120 \mathrm{~h}$ following cell seeding. The number of viable cells was then counted at a wavelength of $450 \mathrm{~nm}$ following the manufacturer's protocol.

Colony formation assay. SGC-7901 human gastric cancer cells were seeded into 6-well plates at a density of 1,000 cells/well and incubated for 12 days. The colonies were fixed with formaldehyde for 20 min then stained with $1 \%$ crystal violet
(Sigma-Aldrich; Merck KGaA) for $30 \mathrm{sec}$. Colonies with over 50 cells were counted.

Flow cytometric analysis of apoptosis. Cells were stained with Annexin V-fluorescein isothiocyanate (Clontech Laboratories, Inc.) and 7-amino-actinomycin D (10 $\mu \mathrm{g} / \mathrm{ml}$; Sigma-Aldrich; Merck $\mathrm{KGaA}$ ) at $4^{\circ} \mathrm{C}$ for $30 \mathrm{~min}$ and were measured using $\mathrm{BD}$ FACSCalibur $^{\mathrm{TM}}$ system (Becton-Dickinson; BD Biosciences). The experiment was repeated three times and the data was analyzed with Multi Cycle Flow System Software (V-321; Phoenix flow systems, Inc.). Cell apoptosis was examined with Annexin V FITC/7-amino-actinomycin D double staining.

Luciferase activity assay. Luciferase activity assay was performed with the Dual-Luciferase Reporter Assay System (Promega Corporation). Cells were seeded into 96-well plates at 4,000 cells/well. The pGL3-luciferase reporter gene plasmids pGL3-CYLD-3'-UTR mutant (Mut) or pGL3-CYLD-3'-UTR wild-type (WT) were purchased from Guangzhou RiboBio Co., Ltd. They were co-transfected (5 $\mu \mathrm{g})$ into SGC-7901 human gastric cancer cells, along with Renilla plasmid and miR-NC (negative control; $100 \mathrm{nmol} / \mathrm{l})$ or miR-454 (100 nmol/l) using Lipofectamine ${ }^{\circledR} 2000$ (Invitrogen; Thermo Fisher Scientific, Inc.). Firefly luciferase activity of the transfection efficiency was normalized to Renilla luciferase activity.

Bioinformatics analysis. The public web-based prediction site TargetScan 7.1 (http://www.targetscan.org) was used to predict the potential miRNA-targeted gene transcripts.

Western blot analysis. Total proteins were extracted with radioimmunoprecipitation assay buffer (1\% NP-40, $0.5 \%$ sodium deoxycholate, $50 \mathrm{mM}$ Tris- $\mathrm{HCl}, 0.1 \%$ SDS, $150 \mathrm{mM}$ $\mathrm{NaCl}, \mathrm{pH} 7.5$ ) supplemented with $1 \%$ protease inhibitor. The solution was centrifuged at $15,000 \mathrm{xg}$ at $4^{\circ} \mathrm{C}$ for $5 \mathrm{~min}$, after which the supernatant was collected. Protein concentrations were quantified using Bio-Rad protein assay (Bio-Rad Laboratories, Inc.). The target protein $(30 \mu \mathrm{g})$ was separated by $10 \%$ sodium dodecyl sulfate polyacrylamide gel electrophoresis and transferred onto the nitrocellulose membranes (Bio-Rad Laboratories, Inc.). The membrane was blocked with $5 \%$ non-fat milk solution for $1 \mathrm{~h}$ at $4^{\circ} \mathrm{C}$ and incubated with anti-GAPDH (1:500; cat. no. ab181602; Abcam) and anti-CYLD (1:1,000; cat. no. 4495; Cell Signaling Technology, Inc.) primary antibodies at $4^{\circ} \mathrm{C}$ for $12 \mathrm{~h}$, washed briefly and then incubated with a horseradish peroxidase-conjugated secondary antibody (1:1,000; cat. no. sc-2357; Santa Cruz Biotechnology, Inc.) at $4^{\circ} \mathrm{C}$ for $2 \mathrm{~h}$. Intensity of bands was analyzed using Image-Pro Plus 6.0 software (Media Cybernetics, Inc.) with chemiluminescence (Amersham Pharmacia Biotech; GE Healthcare) with GAPDH as the loading control.

Statistical analysis. Data are presented as the mean \pm standard deviation. Statistical significance was assessed using unpaired two-tailed Student's t-test for comparisons between two groups or using one-way analysis of variance followed by Newman-Keuls comparison for more than three groups. Data were processed with GraphPad Prism v.8.0 (GraphPad Software, Inc.). $\mathrm{P}<0.05$ was considered to indicate statistical significance. 
A

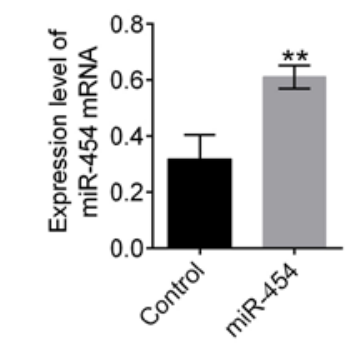

C

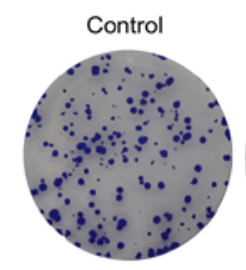

D



B

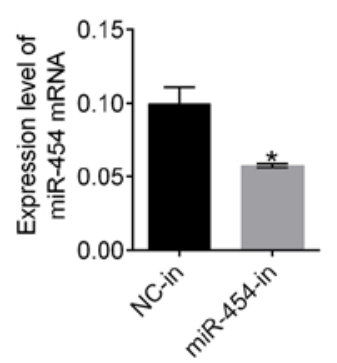


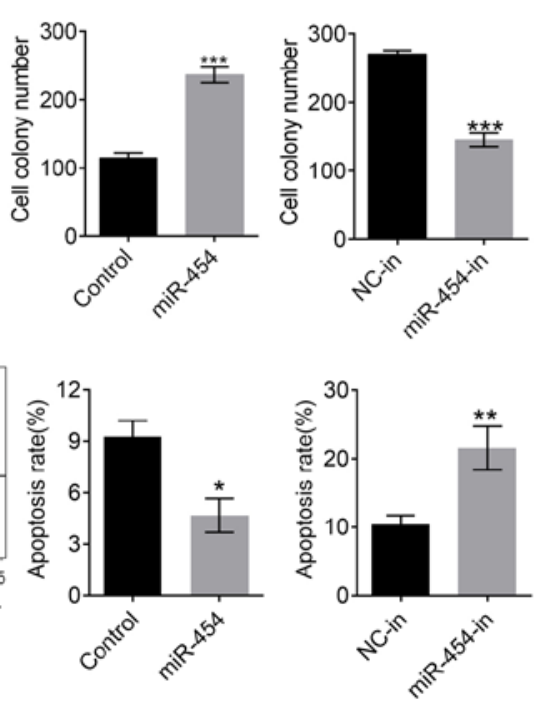

Figure 1. miR-454 promotes proliferation and inhibits apoptosis for SGC-7901 cells. (A) Comparison of miR-454 mRNA expression using RT-qPCR. (B) Proliferation of SGC-7901 cells. (C) Representative colony formation micrographs (left) and quantification of cell colonies (right). (D) Representative flow cytometry analysis (left) and quantification of apoptotic cells (right). ${ }^{*} \mathrm{P}<0.05,{ }^{* *} \mathrm{P}<0.01$ and ${ }^{* * * *} \mathrm{P}<0.001$ vs. control. miR, microRNA; RT-qPCR, reverse transcription-quantitative polymerase chain reaction; OD, optical density; NC, negative control; in, inhibitor.

\section{Results}

miR-454 promotes proliferation and inhibits apoptosis in gastric cancer cells. Expression of miR-454 in SGC-7901 gastric cancer cells was measured post-transfection (Fig. 1A). Compared with the control group, miR-454 expression significantly increased upon transfection $(\mathrm{P}<0.01$; Fig. $1 \mathrm{~A})$ but significantly decreased upon transfection with miR-454 inhibitor ( $\mathrm{P}<0.05$; Fig. 1A). At $120 \mathrm{~h}$, proliferation of the miR-454 groups was significantly higher compared with the control group. Proliferation of the inhibitor-transfected group was significantly lower than the NC-in group $(\mathrm{P}<0.01$; Fig. 1B). The number of colonies of SGC-7901 increased with the expression of miR-454 $(\mathrm{P}<0.001$, Fig. 1C) but decreased in the miR-454-in group compared with the NC-in group ( $\mathrm{P}<0.001$, Fig. $1 \mathrm{C})$. In addition, miR-454 expression was related to SGC-7901 cell apoptosis rate (Fig. 1D) where the control group demonstrated much higher apoptosis rate than the miR-454 group $(\mathrm{P}<0.05$; Fig. 1D) whilst the $\mathrm{NC}$-in group demonstrated a decreased apoptosis rate compared with the miR-454-in group ( $\mathrm{P}<0.01$; Fig. 1D). Taken together, these findings suggested that miR-454 suppressed apoptosis in SGC-7901 cells.

miR-454 directly downregulates CYLD. CYLD was predicted to be a miR-454 target gene, based on analysis with TargetScan (http://www.targetscan.org/vert_72/) (Fig. 2A). The relationship between CYLD and miR-454 was examined by the luciferase reporter assay (Fig. 2B) Results determined that luciferase activity of the wild-type construct was significantly reduced by miR-454 ( $\mathrm{P}<0.05$; Fig. $2 \mathrm{~B})$, but there was no significant effect observed with the mutant construct. These results were further confirmed by data demonstrating that the expression of CYLD mRNA and protein was inhibited by miR-454 overexpression (Fig. 2C and D).

miR-454 promoted proliferation and inhibited apoptosis in gastric cancer cells via targeting CYLD. Western blot analysis determined that the miR-454-induced inhibition of CYLD was reversed upon the transfection of pCDNA CYLD into the SGC-7901 cells (Fig. 3A). Results for the proliferation assays, colony formation and apoptosis assays demonstrated that co-transfection of miR-454 mimic and pCDNA CYLD counteracted the effects of miR-454 on cell proliferation and apoptosis (Fig. 3B-D). These results suggested that the effects of miR-454 on gastric cancer cells were mediated by downregulating CYLD.

miR-454 enhances oxaliplatin resistance in gastric cancer cells. SGC-7901 cells in the miR-454 group exhibited higher cell viability than the control group following treatment with 


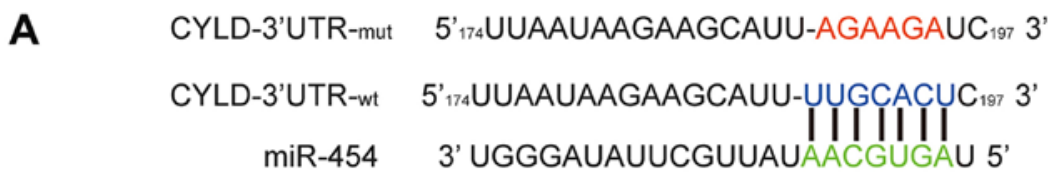
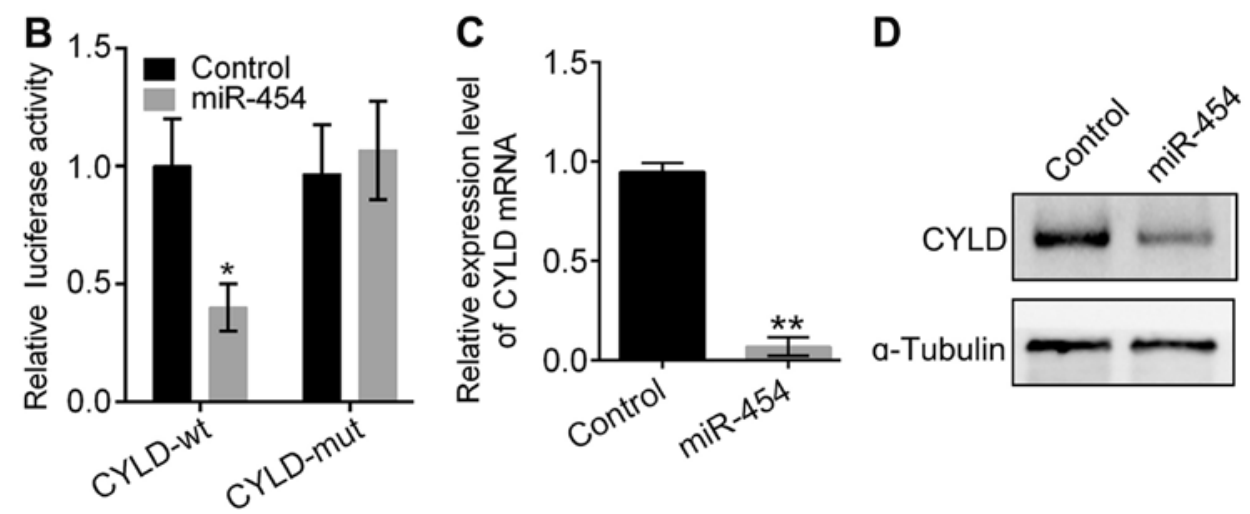

Figure 2. miR-454 directly downregulates CYLD. (A) Predicted target sequence for miR-454 on the 3'UTR of the CYLD gene. Six nucleotides, denoted in red font, were altered to generate the mutant construct. (B) The relative luciferase activities of CYLD-MUT and CYLD-WT. (C) CYLD mRNA expression analysed by RT-qPCR. (D) Western blot analysis of CYLD expression. ${ }^{*} \mathrm{P}<0.05$ and ${ }^{* *} \mathrm{P}<0.01$ vs. control. miR, microRNA; CYLD, cylindromatosis; UTR, untranslated region; MUT, mutant; WT, wild-type; RT-qPCR, reverse transcription-quantitative polymerase chain reaction.

A

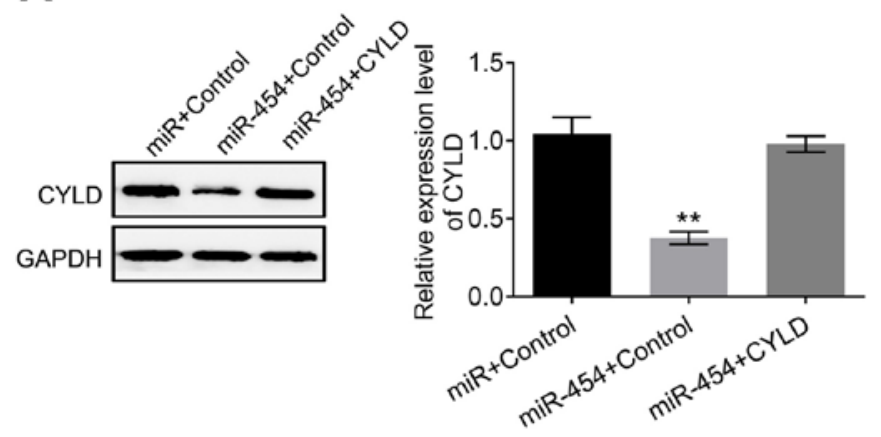

C
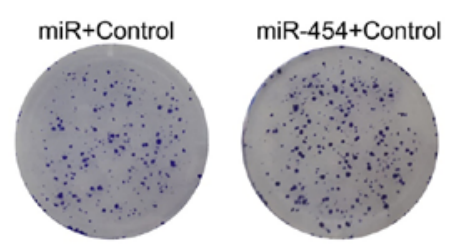

D

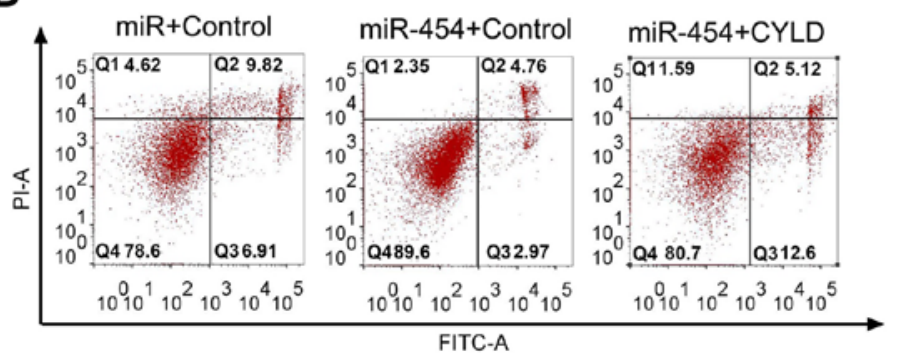

B
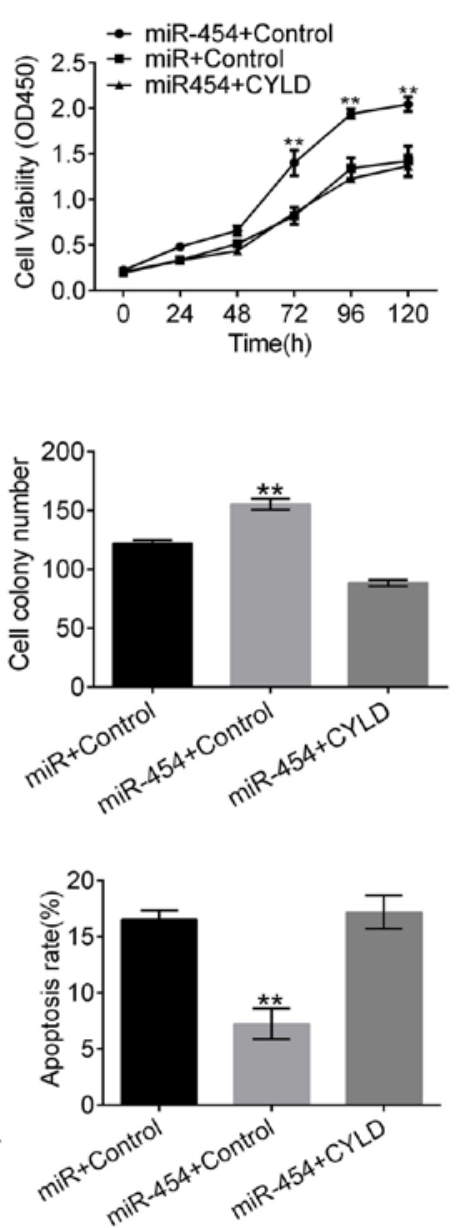

Figure 3. miR-454 promotes proliferation and inhibits apoptosis of SGC-7901 cells via targeting CYLD. (A) Western blot analysis of CYLD expression. (B) Proliferation of SGC-7901 cells. (C) Representative colony formation micrographs (left) and quantification of cell colonies (right). (D) Representative flow cytometry analysis (left) and quantification of apoptotic cells (right). ${ }^{* *} \mathrm{P}<0.01$ vs. control. miR, microRNA; CYLD, cylindromatosis; OD, optical density; FITC, fluorescein isothiocyanate; PI, propidium iodide. 
A

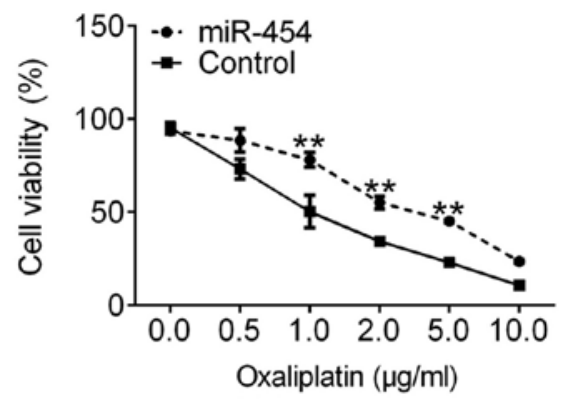

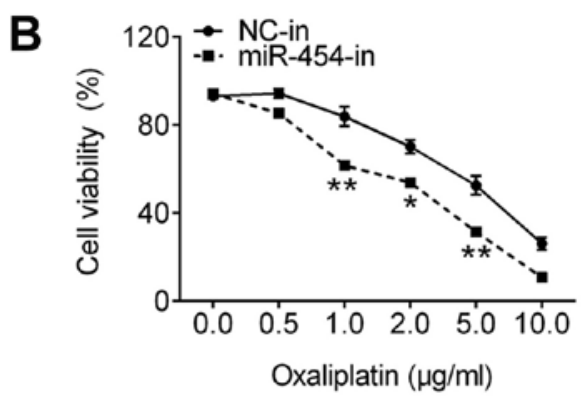
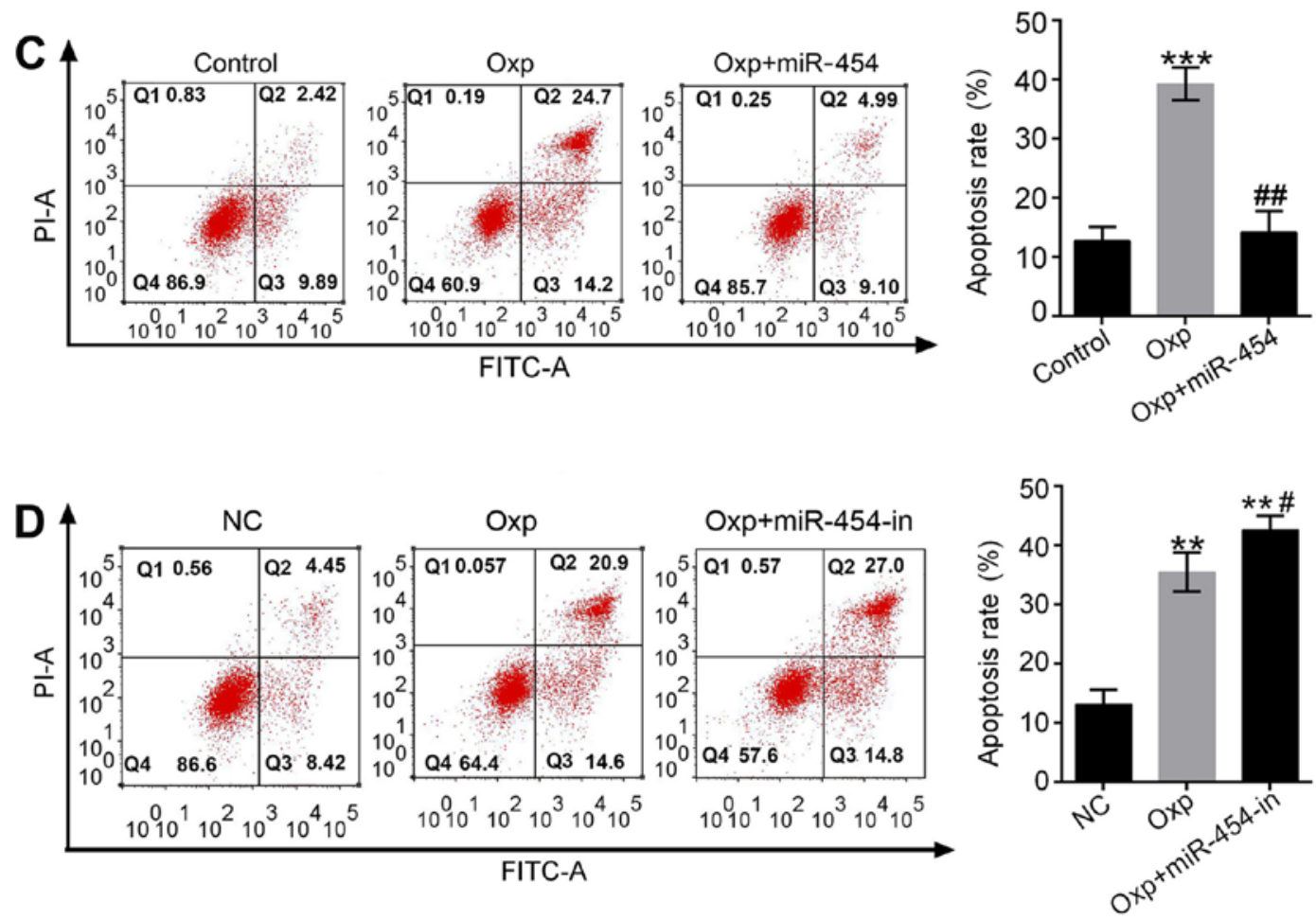

Figure 4. Overexpression of miR-454 increases the resistance to oxaliplatin. (A) Comparisons of cell viability between the control and miR-454 groups with ascending concentrations of oxaliplatin. (B) Comparisons of cell viability between the NC-in and miR-454-in groups with different oxaliplatin concentrations. (C) Representative flow cytometry analysis (left) and quantification of apoptotic cells (right). (D) Representative flow cytometry analysis (left) and quantification of apoptotic cells (right). Cells were collected $48 \mathrm{~h}$ post-transfection for subsequent experimentation. ${ }^{*} \mathrm{P}<0.05$, ${ }^{* *} \mathrm{P}<0.01$ and ${ }^{* * * *} \mathrm{P}<0.001$ vs. control. ${ }^{\#} \mathrm{P}<0.05$ and ${ }^{\# \#} \mathrm{P}<0.01$ vs. oxaliplatin group. miR, microRNA; NC, negative control; in, inhibitor; Oxp, oxaliplatin; FITC, fluorescein isothiocyanate; PI, propidium iodide.

oxaliplatin (Fig. 4A). Cell viability of the miR-454-in group was lower than the $\mathrm{NC}$-in group following oxaliplatin treatment (Fig. 4B). Apoptosis rate was significantly higher for the oxaliplatin group compared with the controls $(\mathrm{P}<0.001$; Fig. 4C). Overexpression of miR-454 reversed the effect of oxaliplatin (Fig. 4C). Apoptosis rates of the oxaliplatin and Oxp + miR-454-in groups increased $(\mathrm{P}<0.01$; Fig. 4D) with the Oxp+miR-454-in group higher than the oxaliplatin group $(\mathrm{P}<0.05$, Fig. 4D). These results suggested that drug resistance was reversed by inhibiting miR-454 and that miR-454 decreased the sensitivity of SGC-7901 cells to oxaliplatin.

\section{Discussion}

The present study evaluated the effects of miR-454 on gastric cancer cell proliferation and explored the corresponding mechanism. The effect of miR-454-induced oxaliplatin resistance was also observed. Overexpression of miR-454 increased the number of gastric cancer cell colonies formed and decreased apoptosis rate, which indicated that miR-454 promoted proliferation. CYLD was directly downregulated by miR-454 thus increasing the survival rate of the SGC-7901 cells. These results suggested that the influence of miR-454 on SGC-7901 cells was achieved via downregulating CYLD. It was also determined that miR-454 reduced the sensitivity of SGC-7901 cells to oxaliplatin, which may further influence the therapeutic effects of oxaliplatin on gastric cancer. The present study provided preliminary evidence for the effect of miR-454 on gastric carcinoma cells and could be used to develop a novel approach for gastric cancer treatment.

Regulation of miRNA expression has recently been used for the treatment of various types of tumor $(16,17)$. The regulatory function of miRNAs in gastric cancer remains not fully understood therefore it would be beneficial to study the role of miR-454 in regards to this condition. Overexpression of miR-454 promoted proliferation of SGC-7901 cells whilst inhibition of miR-454 decreased it which suggested that inhibiting miR-454 may be a potential treatment approach to gastric cancer. 
Effects of miR-454 on the progression of various cancers have been comprehensively reported. Inhibition of miR-454 suppresses the invasion and proliferation of hepatoma cells by inhibiting chromodomain helicase DNA binding protein 5 (14). Tumorigenesis of lung cancer and uveal melanoma is accelerated by miR-454 via targeting the phosphatase and tensin homolog deleted on chromosome ten $(13,10)$. The tumor-suppressing functions of miR-454 have been demonstrated including the inhibition of glioma cell proliferation by miR-454 (11); inhibition of osteosarcoma invasion and proliferation by miR-454 via inhibiting c-Met (12); and cellular radiated susceptibility of renal carcinoma cells promoted by miR-454 via targeting the cell translocation gene (18). The present study demonstrated that miR-454 could regulate the survival of gastric cancer cells.

CYLD is a deubiquitination enzyme that functions as a regulator in the NF- $\kappa \mathrm{B}$ and transforming growth factor- $\beta$ signaling pathways (19), and also as a tumor suppressor (20). It has been reported that CYLD inhibited c-Jun N-terminal kinase and uncontrolled activation of TGF- $\beta$ activated kinase 1 , which, in turn regulated hepatocyte homeostasis $(21,22)$. The present study determined that CYLD was a target gene of miR-454. The results demonstrated that miR-454 downregulated CYLD mRNA and protein expression which increased the apoptosis rate of cancer cells.

The overexpression of miR454 in gastric cancer tissues has been previously reported (23) where it was hypothesized that overexpression of miR-454 was associated with the advancement of the clinical stage, metastases of the lymph node and a poor prognosis for patients. CYLD acts as a suppressor in many types of tumor including breast cancer and non-melanoma skin cancer $(24,25)$. Both miR-425-5p and miR-20a promote the progression of gastric cancer via regulating CYLD $(26,27)$.

Oxaliplatin is an effective chemotherapeutic drug commonly used for treating gastric carcinoma (28). Despite the high remission rate, most patients will ultimately develop resistance to the oxaliplatin-based chemotherapy treatment (29) with the underlying mechanism not fully understood. The present study demonstrated that downregulation of miR-454 could improve the outcome of oxaliplatin treatment on SGC-7901 cells. The in vitro study suggested miR-454 increased stomach cell proliferation and induced drug resistance to oxaliplatin by directly targeting CYLD. It would be of use to carry out in vivo studies to confirm the results, as the in vitro study (i.e., cancer cell lines) might not accurately reflect the in vivo environment however findings demonstrated that miR-454 might be a novel therapeutic target for gastric cancer.

\section{Acknowledgements}

Not applicable.

\section{Funding}

This work was supported by the Science Fund of the National Natural Science Foundation of China (grant no. 81502113) and Wuhan Clinical Research Center for Peritoneal Carcinomatosis (grant no. 2015060911020462).

\section{Availability of data and materials}

The datasets used and/or analyzed during the current study are available from the corresponding author on reasonable request.

\section{Authors' contributions}

$\mathrm{CQH}$ and JYL performed the experiments and wrote the manuscript. XKP collected and analyzed the experimental data. CWP and BX analyzed the experimental data and revised the manuscript. MHF and XJY designed the experiments and approved the final version manuscript. All authors read and approved the final manuscript.

\section{Ethics approval and consent to participate}

Not applicable.

\section{Patient consent for publication}

Not applicable.

\section{Competing interests}

The authors declare that they have no competing interests.

\section{References}

1. Oliveira C, Pinheiro H, Figueiredo J, Seruca R and Carneiro F: Familial gastric cancer: Genetic susceptibility, pathology, and implications for management. Lancet Oncol 16: e60-e70, 2015.

2. AllemaniC,MatsudaT,DiCarlo V,HarewoodR,MatzM,NikšićM, Bonaventure A, Valkov M, Johnson CJ, Estève J, et al: Global surveillance of trends in cancer survival 2000-14 (CONCORD-3): Analysis of individual records for 37513025 patients diagnosed with one of 18 cancers from 322 population-based registries in 71 countries. Lancet 391: 1023-1075, 2018

3. Wadhwa R, Song S, Lee JS, Yao Y, Wei Q and Ajani JA: Gastric cancer-molecular and clinical dimensions. Nat Rev Clin Oncol 10: 643-655, 2013.

4. Goetze OT, Al-Batran SE, Chevallay M and Mönig SP: Multimodal treatment in locally advanced gastric cancer. Updates Surg 70: 173-179, 2018.

5. Bi J, Bai Z, Ma X, Song J, Guo Y, Zhao J, Yi X, Han S and Zhang Z: Txr1: An important factor in oxaliplatin resistance in gastric cancer. Med Oncol 31: 807, 2014.

6. Peng B, Chen Y and Leong KW: MicroRNA delivery for regenerative medicine. Adv Drug Deliv Rev 88: 108-122, 2015.

7. Rupaimoole R and Slack FJ: MicroRNA therapeutics: Towards a new era for the management of cancer and other diseases. Nat Rev Drug Discov 16: 203-222, 2017.

8. El Bezawy R, Cominetti D, Fenderico N, Zuco V, Beretta GL, Dugo M, Arrighetti N, Stucchi C, Rancati T, Valdagni R, et al: miR-875-5p counteracts epithelial-to-mesenchymal transition and enhances radiation response in prostate cancer through repression of the EGFR-ZEB1 axis. Cancer Lett 395: 53-62, 2017.

9. Qiu X and Dou Y: miR-1307 promotes the proliferation of prostate cancer by targeting FOXO3A. Biomed Pharmacother 88 : 430-435, 2017.

10. Zhu DY, Li XN, Qi Y, Liu DL, Yang Y, Zhao J, Zhang CY, Wu K and Zhao S: MiR-454 promotes the progression of human non-small cell lung cancer and directly targets PTEN. Biomed Pharmacother 81: 79-85, 2016.

11. Fang B, Zhu J, Wang Y, Geng F and Li G: MiR-454 inhibited cell proliferation of human glioblastoma cells by suppressing PDK1 expression. Biomed Pharmacother 75: 148-152, 2015.

12. Niu G, Li B, Sun J and Sun L: miR-454 is down-regulated in osteosarcomas and suppresses cell proliferation and invasion by directly targeting c-Met. Cell Prolif 48: 348-355, 2015. 
13. Sun L, Wang QL, Gao XC, Shi DJ, Mi SY and Han Q: MicroRNA-454 functions as an oncogene by regulating PTEN in uveal melanoma. FEBS Lett 589: 2791-2796, 2015.

14. Yu L, Gong XJ, Sun L, Yao H, Lu BL and Zhu LY: miR-454 functions as an oncogene by inhibiting CHD5 in hepatocellular carcinoma. Oncotarget 6: 39225-39234, 2015.

15. Livak KJ and Schmittgen TD: Analysis of relative gene exression data using real-time quantitative PCR and the 2(-Delta Delta C(T)) method. Methods 25: 402-408, 2001

16. Lee HK, Finniss S, Cazacu S, Bucris E, Ziv-Av A, Xiang C, Bobbitt K, Rempel SA, Hasselbach L, Mikkelsen T, et al: Mesenchymal stem cells deliver synthetic microRNA mimics to glioma cells and glioma stem cells and inhibit their cell migration and self-renewal. Oncotarget 4: 346-361, 2013.

17. Yu X, Li Z and Liu J: MiRNAs in primary cutaneous lymphomas. Cell Prolif 48: 271-277, 2015.

18. Wu X, Ding N, Hu WT, He J, Xu S, Pei H, Hua J, Zhou G and Wang J: Down-regulation of BTG1 by miR-454-3p enhances cellular radiosensitivity in renal carcinoma cells. Radiation Oncol 9: 179, 2014.

19. Huang DH, Wang GY, Zhang JW, Li Y, Zeng XC and Jiang N MiR-501-5p regulates CYLD expression and promotes cell proliferation in human hepatocellular carcinoma. Jpn J Clin Oncol 45: 738-744, 2015.

20. Massoumi R: CYLD: A deubiquitination enzyme with multiple roles in cancer. Future Oncol 7: 285-297, 2011.

21. Gautheron $\mathbf{J}$ and Luedde T: A novel player in inflammation and cancer: The deubiquitinase CYLD controls HCC development. J Hepatol 57: 937-939, 2012.

22. Nikolaou K, Tsagaratou A, Eftychi C, Kollias G, Mosialos G and Talianidis I: Inactivation of the deubiquitinase CYLD in hepatocytes causes apoptosis, inflammation, fibrosis, and cancer. Cancer Cell 21: 738-750, 2012.
23. Xu G, Zhu H, Zhang M and Xu J: Histone deacetylase 3 is associated with gastric cancer cell growth via the miR-454-mediated targeting of CHD5. Int J Mol Med 41: 155-163, 2018.

24. Masoumi KC, Shaw-Hallgren G and Massoumi R: Tumor suppressor function of CYLD in nonmelanoma skin cancer. J Skin Cancer 2011: 614097, 2011.

25. Orfanidou T, Xanthopoulos K, Dafou D, Pseftogas A, Hadweh P, Psyllaki C, Hatzivassiliou E and Mosialos G: Down-regulation of the tumor suppressor CYLD enhances the transformed phenotype of human breast cancer cells. Anticancer Res 37: 3493-3503, 2017.

26. Yan YF, Gong FM, Wang BS and Zheng W: MiR-425-5p promotes tumor progression via modulation of CYLD in gastric cancer. Eur Rev Med Pharmacol Sci 21: 2130-2136, 2017.

27. Zhu M, Zhou X, Du Y, Huang Z, Zhu J, Xu J, Cheng G, Shu Y, Liu P, Zhu W and Wang T: miR-20a induces cisplatin resistance of a human gastric cancer cell line via targeting CYLD. Mol Med Rep 14: 1742-1750, 2016.

28. Niu J and Mims MP: Oxaliplatin-induced thrombotic thrombocytopenic purpura: Case report and literature review. J Clin Oncol 30: E312-E314, 2012.

29. Tsimberidou AM, Said R, Culotta K, Wistuba I, Jelinek J, Fu S, Falchook G, Naing A, Piha-Paul S, Zinner R, et al: Phase I study of azacitidine and oxaliplatin in patients with advanced cancers that have relapsed or are refractory to any platinum therapy. Clin Epigenetics 7: 29, 2015.

This work is licensed under a Creative Commons Attribution-NonCommercial-NoDerivatives 4.0 International (CC BY-NC-ND 4.0) License. 